ARTÍCULO ORIGINAL

\title{
EFECTO HIPOGLICEMIANTE DE Moringa oleifera (MORINGA) COMPARADO CON Smallanthus sonchifolius (YACÓN) EN Rattus norvegicus CON DIABETES MELLITUS INDUCIDA
}

\author{
Otto W. Vargas-Tineo (1,a, Dina M. Segura-Muñoz $\mathbb{1}^{1, a}$, Lizzie K. Becerra-Gutiérrez ${ }^{11,2, b}$, \\ José P. Amado-Tineo (10) ${ }^{3, c}$, Heber Silva-Díaz (10) ${ }^{1,2, b}$ \\ 1 Facultad de Medicina Humana, Universidad de San Martín de Porres, Chiclayo, Perú. \\ 2 Dirección de Investigación, Hospital Regional Lambayeque, Chiclayo, Perú. \\ 3 Facultad de Medicina, Universidad Nacional Mayor de San Marcos, Lima, Perú. \\ a Estudiante de Medicina Humana; ${ }^{b}$ biólogo, doctor en Ciencias; ${ }^{c}$ médico cirujano, especialista en Medicina Interna, doctor \\ en Medicina.
}

\section{RESUMEN}

Objetivo: Comparar el efecto hipoglicemiante del extracto acuoso de Moringa oleifera (moringa), Smallanthus sonchifolius (yacón) y metformina en Rattus norvegicus, variedad albina, con diabetes mellitus inducida. Materiales y métodos: Estudio preclínico, experimental controlado y aleatorizado. La diabetes se indujo por vía intraperitoneal con una dosis de aloxano a $130 \mathrm{mg} / \mathrm{kg}$ de peso vivo (PV); se emplearon 24 Rattus norvegicus, variedad albina, machos, cepa Holfzman (seis por grupo). Se dividieron de la siguiente manera: grupo control (sin tratamiento), grupo metformina (14 mg/kg PV), grupo M. oleifera (200 mg/ $\mathrm{kg} \mathrm{PV}$ ), y grupo S. sonchifolius (140 mg/kg PV), los tratamientos fueron administrados mediante sonda orogástrica durante 15 días. Los niveles de glicemia fueron determinados usando un glucómetro electrónico Accu-Chek ${ }^{\circledR}$ Instant (Roche). Resultados: Se observó reducción de la glicemia en los tratamientos: M. oleifera ( $\mathrm{p}=0,009)$, S. sonchifolius $(\mathrm{p}=0,002)$ y metfotmina $(\mathrm{p}=0,002)$, en $313 \mathrm{mg} / \mathrm{dL}, 281,5 \mathrm{mg} / \mathrm{dL}$ y $415 \mathrm{mg} / \mathrm{dL}$, respectivamente. En cuanto a la comparación de la glicemia en los grupos tratados y control, se observó que a las 24 horas y cuatro días de tratamiento no hubo diferencia $(p>0,05)$; mientras que al octavo $(p<0,05)$ y décimo quinto día $(\mathrm{p}<0,01)$ los grupos tratados tuvieron menor glicemia respecto al control, pero similares entre ellos. Conclusión: El extracto acuoso de S. sonchifolius y de M. oleifera, y la metformina presentaron similar efecto hipoglicemiante en ratas de experimentación con diabetes inducida.

Palabras clave: Hipoglicemiante; Diabetes Mellitus; Moringa oleífera; Ratas; Aloxano (fuente: DeCS BIREME).

Citar como: Vargas-Tineo OW, SeguraMuñoz DM, Becerra-Gutiérrez LK, Amado-Tineo JP, Silva-Díaz H. Efecto hipoglicemiante de Moringa oleifera (moringa) comparado con Smallanthus Sonchifolius (yacón) en Rattus norvegicus con diabetes mellitus inducida. Rev Peru Med Exp Salud Publica. 2020;37(3):47884. doi: https://doi.org/10.17843/ rpmesp.2020.373.5275

Correspondencia: Heber Silva Díaz; Av. Los Eucaliptos 300-304, Urb. La Pradera, distrito de Pimentel, Chiclayo, Perú:

h.silvadiaz185@gmail.com

Recibido: $18 / 02 / 2020$ Aprobado: $24 / 06 / 2020$

En línea: $10 / 08 / 2020$

\section{HYPOGLYCEMIC EFFECT OF Moringa oleifera (MORINGA) COMPARED WITH Smallanthus sonchifolius (YACON) ON Rattus norvegicus WITH INDUCED DIABETES MELLITUS}

\section{ABSTRACT}

Objective: To compare the hypoglycemic effect of the aqueous extract of Moringa oleifera (moringa), Smallanthus sonchifolius (yacon) and metformin on Rattus norvegicus, albino variety, with induced diabetes mellitus. Materials and methods: Preclinical, experimental, controlled and randomized study. Diabetes was induced intraperitoneally with a dose of alloxan at $130 \mathrm{mg} / \mathrm{kg}$. A total of 24 male Rattus norvegicus, albino variety, Holfzman strain (6 per group) were used. They were divided as follows: control group (no treatment), metformin group (14 mg/kg), M. oleifera group $(200 \mathrm{mg} / \mathrm{kg})$, and S. sonchifolius group $(140 \mathrm{mg} / \mathrm{kg})$, treatments were administered via orogastric tube for 15 days. Glycemia levels were determined using an Accu-Chek Instant electronic glycometer (Roche). Results: Decreased glycemia was observed in the treatment groups: M. oleifera $(\mathrm{p}=0.009)$, S. sonchifolius $(\mathrm{p}=0.002)$ and metformin $(\mathrm{p}=0.002)$, by $313 \mathrm{mg} / \mathrm{dL}, 281.5 \mathrm{mg} / \mathrm{dL}$ and $415 \mathrm{mg} / \mathrm{dL}$, respectively. When comparing glycemia in the treated and control groups, no difference was observed $(\mathrm{P}>0.05)$ at 24 hours and four days of treatment; while at the eighth $(\mathrm{P}<0.05)$ and fifteenth day $(\mathrm{P}<0.01)$ the treated groups had lower glycemia than the control group, but it was similar among them. Conclusion: The aqueous extract of $S$. sonchifolius, $M$. oleifera, and metformin presented similar hypoglycemic effect in experimental rats with induced diabetes.

Keywords: Hypoglycemic Agents; Diabetes Mellitus; Moringa oleifera; Rats; Alloxan (source: MeSH NLM). 


\section{INTRODUCCIÓN}

La Organización Mundial de la Salud sostiene que el número de personas con diabetes mellitus (DM) en el mundo ha aumentado de 108 millones en 1980 a 422 millones en 2014 y que será la séptima causa de defunción para $2030^{(1,2)}$. La diabetes mellitus tipo 2 (DM 2) es un problema en crecimiento, 371 millones de adultos viven con este tipo de diabetes en el mundo, de los cuales 26 millones (7\%) residen en Latinoamérica ${ }^{(3)}$. El Instituto Nacional de Estadística e Informática de Perú (INEI) informó que a nivel nacional el 3,9\% de la población de 15 a más años fue diagnosticada con DM en $2019^{(4)}$. En la costa del Perú se reportó mayor población con diabetes $(4 \%)$ y mayor incidencia en la zona urbana ${ }^{(5)}$.

La DM es una enfermedad crónica degenerativa, sus complicaciones crónicas más frecuentes son la nefropatía, neuropatía, retinopatía, cetoacidosis y la enfermedad cardiovascular ${ }^{(6)}$. El tratamiento de la DM 2 es multifactorial y personalizado, teniendo como pilares la nutrición, la actividad física y medicamentos con reportes de adherencia del $47,8 \%{ }^{(7)}$. Las guías internacionales recomiendan como primera línea de tratamiento farmacológico a la metformina ${ }^{(8)}$.

Las plantas medicinales (PM) constituyen una alternativa terapéutica viable debido a su bajo costo y fácil disponibilidad para muchas poblaciones, más de 400 PM con gran diversidad fitoquímica son estudiadas por su potencial antidiabético y menor o ningún efecto secundario, por lo que es importante validar científicamente la efectividad y seguridad para garantizar su uso ${ }^{(9-13)}$. La Moringa oleifera (moringa) y Smallanthus sonchifolius (yacón) son PM de fácil acceso y bajo costo en nuestro medio.

La Moringa oleifera es un árbol originario del sur del Himalaya ${ }^{(14)} \mathrm{y}$ forma parte de la familia Moringaceae ${ }^{(15)}$. Las hojas de la M. oleifera, además de proteínas, minerales y vitaminas, contienen los siguientes principios fitoquímicos: flavonoides, ácidos fenólicos, alcaloides y carotenoides, isotiocianatos, glucosinolatos y taninos, saponinas, oxalatos y fitatos ${ }^{(13)}$. Se han identificado posibles compuestos con efecto hipoglicemiante $\mathrm{y}$ antioxidante en estudios fitoquímicos previos ${ }^{(6)}$, que actúan por diferentes mecanismos en los que incluyen la inhibición de las actividades de $\alpha$-amilasa y $\alpha$-glucosidasa, aumento de la captación de glucosa en los músculos y el hígado, inhibición de la captación de glucosa desde el intestino, disminución de la gluconeogénesis en el hígado y aumento de la secreción y sensibilidad a la insulina ${ }^{(13)}$. Asimismo, los estudios revisados de toxicidad en animales de experimentación han mostrado que los extractos acuosos y alcohólicos de la $M$. oleifera no tienen efectos adversos ${ }^{(13)}$. Además, la M. oleifera protege los tejidos del estrés oxidativo ${ }^{(16)}$, reduce la actividad de los radicales libres, la peroxidación lipídica y previene el desarrollo de complicaciones crónicas ${ }^{(6)}$.

El S. sonchifolius pertenece a la familia Asteraceae, originario de los valles andinos de Sudamérica; se cultiva a altitudes de 2000

\section{MENSAJES CLAVE}

Motivación para realizar el estudio: El incremento de la población peruana con diagnóstico de diabetes y la búsqueda de nuevos principios activos con efecto hipoglicemiantes útiles para su tratamiento.

Principales hallazgos: El extracto acuoso del Smallanthus sonchifolius (yacón) y de la Moringa oleifera (moringa), y metformina presentaron similar efecto hipoglicemiante en ratas de experimentación con diabetes inducida por aloxano.

Implicancias: La moringa y el yacón tuvieron efecto hipoglicemiante similar a la metformina en un modelo animal. Se requiere realizar estudios preclínicos con principios activos derivados de estas plantas, y posteriores estudios clínicos.

a $3100 \mathrm{~m}$ s. n. m. y alcanza la madurez entre 6 y 12 meses después de la siembra ${ }^{(17)}$. La composición fitoquímica del S. sonchifolius ha sido descrita previamente y ha revelado altas concentraciones de grasas y aceites, fenoles y taninos, alcaloides, lactonas, flavonoides y antocianidinas ${ }^{(12)}$; asimismo, se ha reportado su alta seguridad en pruebas de toxicidad aguda en modelos experimetnales, tanto de sus extractos atomizados como de sus alcaloides ${ }^{(12)}$. El S. sonchilfolius tiene efectos hipoglicemiantes, induce la liberación de insulina y aumenta su concentración en el plasma en ratas diabéticas y normales ${ }^{(18)}$. Este efecto ha sido confirmado principalmente por el ácido cafeico y clorogénico junto con tres ácidos dicaffeoilquinicos isoméricos, que podrían contribuir satisfactoriamente a una inhibición de la $\alpha$-glucosidasa en el borde en cepillo de las células del intestino delgado. La decocción de hojas de yacón mostró in vitro e in vivo alargar el tiempo de absorción de la glucosa, retrasando la digestión rápida de la sacarosa; un componente aislado del yacón, la lactona sesquiterpénica disminuye significativamente los niveles de glicemia posprandial en ratas diabéticas ${ }^{(19)}$.

Se ha demostrado el efecto hipoglicemiante in vitro de estas PM ${ }^{(12,13,18,19)}$; sin embargo, se desconoce cuál de las dos plantas tiene mayor efecto in vivo, lo que permitiría considerarlas como una alternativa de tratamiento en DM, después de los posteriores y correspondientes ensayos clínicos. El objetivo del presente estudio fue comparar el efecto hipoglicemiante del extracto acuoso de la $M$. oleifera, el de $S$. sonchifolius, y la metformina en $R$. norvegicus con diabetes mellitus inducida.

\section{MATERIALES Y MÉTODOS}

Diseño de estudio y animales de experimentación Se realizó un estudio experimental controlado y aleatorizado con ratas albinas (Rattus norvegicus, variedad albina) 
machos que desarrollaron diabetes mellitus inducida con aloxano. Se excluyeron todas aquellas que no elevaron sus niveles de glucosa (como mínimo $250 \mathrm{mg} / \mathrm{dL}$ ) y que presentaron alguna patología.

Se seleccionaron 24 machos de la cepa Holfzman, de entre 12 y 14 semanas de edad aproximadamente y con un peso de $180 \pm 20 \mathrm{~g}$. Los animales fueron adquiridos en el bioterio del Hospital Regional Lambayeque, donde tuvieron diez días de adaptación con comida balanceada procedente de la Universidad Nacional Agraria La Molina (UNALM). La dieta recibida fue de crecimiento con el siguiente valor nutricional: agua, $10 \%$; proteínas, $13-14 \%$; lípidos, $3-4 \%$; y calcio, $0,5 \%$; y según la hoja del fabricante, fue elaborado con los siguientes ingredientes: maíz amarillo, torta de soya $48 \%$, pasta de algodón, torta de girasol, subproductos de agroindustria, heno de alfalfa, carbonato de calcio, fosfato dicálcico, aminoácidos sintéticos, promotores orgánicos, vitaminas, minerales, cloruro de sodio, antifúngico y antioxidantes.

\section{Procedimiento}

\section{Recolección y secado de materiales vegetales}

Las hojas de moringa se recolectaron del caserío Yurimaguas, distrito de Jayanca de la provincia de Lambayeque, de aproximadamente dos meses de sembrado. Las hojas de yacón se recolectaron en el caserío Montegrande Bajo, distrito de Huarmaca en la provincia de Huancabamba, Piura, de aproximadamente siete meses de sembrado. Las muestras fueron depositadas en el Herbario Lambayeque PRG, del departamento de Botánica de la Universidad Nacional Pedro Ruiz Gallo (UNPRG) e identificadas por un especialista calificado.

Las hojas de moringa y yacón fueron lavadas y puestas en un secador de bandeja con aire caliente durante dos horas a $56{ }^{\circ} \mathrm{C}$, se separaron tallos de hojas, para luego triturar solo hojas con ayuda de un mortero (cada planta por separado). Los triturados de cada planta fueron almacenados en recipientes herméticos por separado, aislados de luz y humedad.

\section{Preparación de tratamientos}

Preparación del extracto acuoso de M. oleifera y S. sonchifolius a $100 \mathrm{mg} / \mathrm{ml}$

En el Laboratorio de Investigación del Hospital Regional Lambayeque (HRL) se pesó $10 \mathrm{~g}$ de moringa y de yacón con la ayuda de una balanza analítica; posteriormente, en una cabina de bioseguridad, se mezclaron con $100 \mathrm{~mL}$ de agua destilada estéril, se colocaron en tubos estériles de $15 \mathrm{~mL}$, luego llevados a baño maría por 30 minutos a $90{ }^{\circ} \mathrm{C}$. Posteriormente, se centrifugó a 3500 RPM durante diez minutos. En la cabina de bioseguridad, se extrajo el sobrenadante con ayuda de una jeringa estéril de $10 \mathrm{~mL}$ que constituyó el extracto acuoso. Se prepararon alícuotas y se conservaron entre 2 y $8{ }^{\circ} \mathrm{C}$ hasta su uso en los siguientes próximos cuatro días.
Preparación de metformina a $10 \mathrm{mg} / \mathrm{mL}$

En el Laboratorio de Investigación se pesó $1 \mathrm{~g}$ de metformina (Sigma, USA) y se diluyó en $100 \mathrm{~mL}$ de agua destilada ${ }^{(20)}$, se homogenizó y conservó a $2-8^{\circ} \mathrm{C}$ hasta su uso en los siguientes próximos cuatro días.

Inducción de diabetes mellitus en Rattus norvegicus variedad albina cepa Holfzman

Se les indujo diabetes experimental con una única dosis de aloxano (Sigma, St. Louis, MI, EUA), a dosis de $130 \mathrm{mg} / \mathrm{kg}$ de peso vivo (PV) vía intraperitoneal. Los niveles de glicemia se midieron a las 72 horas ${ }^{(21)}$ y se consideraron diabéticas, aquellas que tengan un nivel de glicemia mayor de $250 \mathrm{mg} / \mathrm{dL}^{(22)}$.

\section{Distribución de grupos de estudio}

A las ratas diabéticas se las distribuyó en cuatro grupos de seis ratas cada uno. Se enumeró cada rata con un marcador indeleble y se asignaron aleatoriamente con el programa Epidat 4.1. Estos fueron los grupos: grupo control, el cual no recibió tratamiento, y bebió agua a voluntad; grupo metformina, que recibió $14 \mathrm{mg} / \mathrm{kg}$ PV, tratamiento estándar de DM $2^{(20)}$; grupo M. oleifera, que recibió $200 \mathrm{mg} / \mathrm{Kg} \mathrm{PV}$ de extracto acuoso de M. oleifera ${ }^{(16)}$; y grupo S. sonchifolius, que recibió $140 \mathrm{mg} / \mathrm{Kg}$ PV de extracto acuoso de S. sonchifolius ${ }^{(19)}$.

La evaluación y duración del experimento se llevó a cabo durante 15 días consecutivos. Se realizó la medición de los niveles de glicemia a las siete horas. Asimismo, las sustancias con efecto hipoglicemiante fueron administradas a las ocho horas, mediante una sonda orogástrica descartable (vía orogástrica), salvo en el grupo control que no recibió tratamiento.

A todos los animales de experimentación se les dio una ración de $14 \mathrm{~g}$ de alimento balanceado para roedores una vez al día posterior al tratamiento correspondiente. El día anterior a la medición de la glicemia se mantuvo la comida en sus jaulas a las diez y a las 19 horas, luego se les retiró para el ayuno de 12 horas con agua ad libitum.

\section{Medición de la glicemia en ratas}

La concentración de glucosa en sangre se midió con el glucómetro Accu-Chek Instant (Roche). El nivel de glicemia durante el experimento fue monitoreado al primer, cuarto, octavo y décimo quinto día postratamiento. Para realizar este procedimiento, los especímenes permanecieron en ayunas 12 horas aproximadamente, se recolectó la muestra de sangre por punción en el ápice de las colas, previa antisepsia del área con alcohol al 70\%, desechando la primera gota y recibiendo la siguiente sobre la tira reactiva. Los valores obtenidos se expresaron en miligramos por decilitro $(\mathrm{mg} / \mathrm{dL})$.

Según el reporte del fabricante, el sistema Accu-Chek ${ }^{\circ}$ Instant (medidor y tiras) se calibra en fábrica a partir de sangre capilar de personas diabéticas (comparación de métodos y exactitud), sangre venosa (repetibilidad) y solución de control (reproducibilidad). Asimismo, al inicio y a la mitad 
de las mediciones en las ratas, se realizaron mediciones con la solución control administrada por el fabricante, que contenía una concentración de glucosa de $100 \mathrm{mg} / \mathrm{dL}$. El equipo cumple con los requerimientos establecidos por las normas ISO 15197:2013 e ISO 15197:2015 para sistemas de ensayo para diagnóstico in vitro, requisitos de los sistemas de autodiagnóstico de monitoreo de glucosa en sangre en el manejo de la $\mathrm{DM}^{(23)}$.

\section{Análisis estadístico}

Los datos se codificaron y digitaron en Microsoft Excel 2016 y se procesaron con IBM SPSS 24 (IBM Corp., Armonk, N.Y., EUA). Luego del análisis de la curva de distribución y el resultado de la prueba de normalidad de Shapiro-Wilks, se realizó un análisis descriptivo de la glicemia en todos los tratamientos y mediciones, mediante el cálculo de medianas y rangos intercuartílicos.

El análisis comparativo del efecto hipoglicemiante de los extractos de M. oleifera, de S. sonchifolius, y metformina, se realizó mediante las pruebas no paramétricas de Kruskall Wallis y test de Dunn (comparaciones múltiples). Asimismo, para medir la reducción de la glicemia pretratamiento respecto al décimo quinto día de tratamiento se utilizó la prueba no paramétrica de Wilcoxon. Se consideró significativo un valor de $\mathrm{p}<0,05$.

\section{Aspectos éticos}

La investigación se realizó respetando los principios de reducir el número de animales experimentales, reemplazar los animales de experimentación por otros métodos y refinar las técnicas para aminorar el sufrimiento. El protocolo fue revisado y aprobado por el Comité de Ética en Investigación para Uso de Animales (CEIPUA), del HRL. Asimismo, se respetaron los principios y aspectos relacionados con el cuidado y uso de animales de laboratorio detallados en la Directiva 2010/63/UE del Parlamento Europeo y del Consejo, respecto a la protección de los animales utilizados para fines científicos, y la Ley Nacional de Protección de Animales en Cautiverio ${ }^{(24)}$. Al final del experimento las ratas fueron eutanasiadas con pentobarbital sódico a dosis de $100 \mathrm{mg} / \mathrm{kg} \mathrm{PV}$.

\section{RESULTADOS}

Los datos tuvieron distribución no normal. En la Tabla 1, se muestran medianas y rangos intercuartílicos de la glicemia de ratas con diabetes mellitus inducida, distribuida según grupos experimentales (control y tratamientos con M. oleifera, S. sonchifolius, y metformina).

Se observó reducción de la glicemia en los tratamientos con metformina, $M$. oleifera y $S$. sonchifolius, en $415 \mathrm{mg} / \mathrm{dL}$, $313 \mathrm{mg} / \mathrm{dL}$ y 281,5 mg/dL; respectivamente (Tabla 2).

En la Figura 1, se visualiza la tendencia de la glicemia en los grupos experimentales, donde se observa que a medida que transcurre el tiempo, la glicemia de los tratamientos disminuye en comparación al control, quienes no recibieron tratamiento.

En cuanto a la comparación de las glicemias en los grupos tratados y control, se observó que a las 24 horas y cuatro días de tratamiento no hubo diferencia significativa $(p>0,05)$; mientras que al octavo y décimo quinto día, los tratamientos tuvieron una menor glicemia respecto al control, siendo esta diferencia estadísticamente significativa (Tabla 3 y 4 ).

\section{DISCUSIÓN}

Se encontró que los extractos acuosos de S. sonchifolius y M. oleifera tuvieron efecto hipoglicemiante significativo, similar al comprobado con metformina. Al respecto, estudios previos han descrito que los extractos acuosos de hojas de yacón y moringa son frecuentemente utilizados en humanos por sus múltiples beneficios para la salud, entre los que resalta la reducción de la glucosa postprandial ${ }^{(18-20)}$.

Asimismo, otro estudio encontró que la decocción de las hojas de yacón contiene compuestos fenólicos desde cafeico, clorogénico y ácidos dicaffeoilquinicos, el ácido ferúlico, el ácido pcumárico, el ácido protocatechuico y la quercetina contribuirían en su efecto hipoglicemiante inhibiendo la a-glucosidasa, promoviendo la regulación de la glucosa ${ }^{(19,25)}$. Su acción hipoglicemiante también es atribuida por unirse a los receptores de insulina y potenciar la actividad de la en-

Tabla 1. Glicemia en ratas con diabetes mellitus inducida, 15 días postratamiento con extracto de $140 \mathrm{mg} / \mathrm{kg}$ PV Smallanthus sonchifolius (yacón), $200 \mathrm{mg} / \mathrm{kg}$ PV Moringa oleifera (moringa) y metformina $14 \mathrm{mg} / \mathrm{kg}$ PV

\begin{tabular}{|c|c|c|c|c|c|}
\hline \multirow[b]{2}{*}{ Grupos $(n=6)$} & \multicolumn{5}{|c|}{ Glicemia (mg/dL) } \\
\hline & $\begin{array}{c}\text { Pre Tto } \\
\text { Me (RIC) }\end{array}$ & $\begin{array}{c}1 \text { dTto } \\
\text { Me (RIC) }\end{array}$ & $\begin{array}{c}4 \text { dTto } \\
\text { Me (RIC) }\end{array}$ & $\begin{array}{r}8 \text { dTto } \\
M e(\text { RIC) }\end{array}$ & $\begin{array}{c}15 \text { dTto } \\
\text { Me (RIC) }\end{array}$ \\
\hline Control & $439(315-565)$ & $402(166-469)$ & $445,5(402-600)$ & $586,5(522-600)$ & $389(330-512)$ \\
\hline Metformina & $522(471-600)$ & $331(191-476)$ & $271(77-412)$ & $127(115-251)$ & $107(99-119)$ \\
\hline M. oleifera & $417(341-600)$ & $451(378-600)$ & $427(417-457)$ & $246(170-421)$ & $110(104-170)$ \\
\hline S. sonchifolius & $398(353-471)$ & $268(249-435)$ & $298(182-361)$ & $134(121-346)$ & $117(101-232)$ \\
\hline
\end{tabular}

Tto: Tratamiento; dTto: días con tratamiento; Me: mediana; RIC: rango intercuartílico 
Tabla 2. Reducción de la glicemia en ratas con diabetes mellitus inducida y tratadas con extracto de $140 \mathrm{mg} / \mathrm{kg}$ PV Smallanthus sonchifolius (yacón), 200 mg/kg PV Moringa oleifera (moringa) y metformina 14 mg/kg PV

\begin{tabular}{|c|c|c|c|c|c|}
\hline \multirow{2}{*}{ Grupos $(n=6)$} & \multicolumn{2}{|c|}{ Mediana } & \multicolumn{2}{|c|}{ Disminución } & \multirow{2}{*}{ Valor de $\mathbf{p}^{\star}$} \\
\hline & Pre Tto & 15 dTto & $\mathrm{mg} / \mathrm{dL}$ & $\%$ & \\
\hline Control & 439,0 & 389,0 & 50,0 & 11,4 & 0,981 \\
\hline Metformina & 522,0 & 107,0 & 415,0 & 79,5 & 0,002 \\
\hline M. oleifera & 423,0 & 110,0 & 313,0 & 74,0 & 0,009 \\
\hline S. sonchifolius & 398,0 & 116,5 & 281,5 & 70,7 & 0,002 \\
\hline
\end{tabular}

Tto: Tratamiento; dTto: días con tratamiento

${ }^{*}$ Prueba de Wilcoxon.

zima tirosinaquinasa cuya finalidad es disminuir los niveles de glucosa, además de proteger frente a las complicaciones producidas por la diabetes ${ }^{(18)}$.

El efecto hipoglicemiante del extracto acuoso de yacón observado en nuestro estudio fue similar al reportado por Dos Santos et al., donde obtuvo descensos cercanos a la normalidad de los niveles de glucosa en ratas tratadas con extractos hidroetanólicos ${ }^{(25)}$. Asimismo, Mejía et al. estudiaron el efecto hipoglicemiante usando raíz entera de yacón, obteniendo una menor disminución de los niveles de glucosa $(27,6 \mathrm{mg} / \mathrm{dl}$ en 34 días) ${ }^{(26)}$, comparado con nuestro estudio donde obtuvimos una disminución de $281,5 \mathrm{mg} / \mathrm{dL}$ en 15 días.

De igual manera, el grupo tratado con $M$. oleifera disminuyó significativamente los niveles de glicemia en comparación con el control, este resultado pudo haberse debido a su alto contenido de polifenoles y compuestos flavonoides ${ }^{(6,13)}$, glucosinolatos e isotiocianatos ${ }^{(27)}$, terpenoides, quercetina y kaempferol ${ }^{(28)}$ encontrados en estudios fitoquímicos anteriores, donde actúan como secretagogos de insulina y contribuyen atenuando las complicaciones diabéticas ${ }^{(16,28)}$ ya que, mejoran la regeneración y viabilidad de las células destruidas; otro de sus mecanismos es reducir la gluconeogénesis y glucogenólisis hepáticas atribuido al ácido clorogénico contenido en sus hojas ${ }^{(28)}$.

Nuestros resultados son congruentes con los obtenidos por estudios previos donde ratas de experimentación hiper-

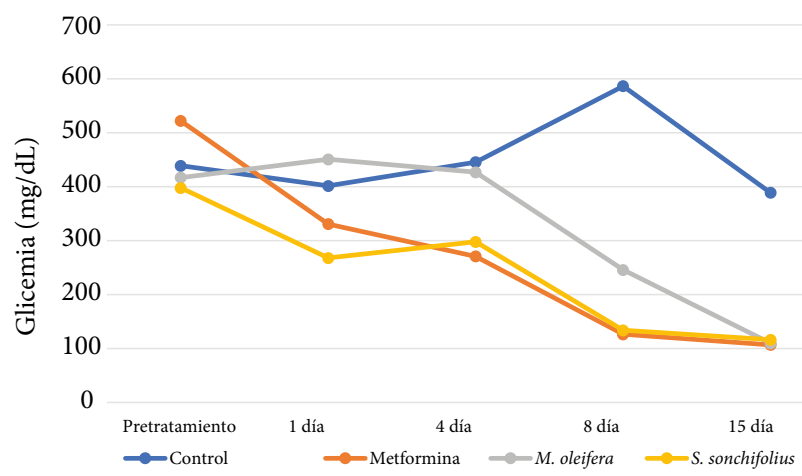

Figura 1. Glicemia en ratas con diabetes mellitus inducida, tratadas con $140 \mathrm{mg} / \mathrm{kg}$ PV de extracto de S. sonchifolius (yacón), $200 \mathrm{mg} / \mathrm{kg}$ PV de $M$. oleifera (moringa) y $14 \mathrm{mg} / \mathrm{kg}$ PV de metformina. glucémicas son tratadas con extracto de $M$. oleifera obteniendo una disminución significativa de glicemia ${ }^{(6,20,29)}$.

Los extractos acuosos de M. oleifera y $S$. sonchifolius tuvieron similar efecto hipoglicemiante sin diferencia significativa, esto pudo deberse a que ambas plantas contienen similitud en su composición como son los polifenoles, a quienes se les atribuye propiedades hipoglicemiantes ${ }^{(19,25,27,28)}$, aunque todavía no se ha establecido exactamente su mecanismo de acción, que parece ser a varios niveles.

Se observó reducción de la glicemia pretratamiento con respecto al décimo quinto día de tratamiento en el control positivo (79,5\%), tratamientos con $M$. oleifera (74\%) y $S$. sonchifolius (70,7\%). Estudios previos informan que grupos tratados con $M$. oleifera obtuvieron valores similares al efecto hipoglicemiante producido por la metformina ${ }^{(20,29)}$. Pero no hay reportes similares con yacón y tampoco se encontraron estudios que comparen ambas PM.

El reducido tamaño de muestra fue una limitante del presente estudio, sin embargo, es usual en este tipo de diseños por aspectos éticos y logísticos, a pesar de ello, consideramos que los resultados son válidos ${ }^{(19,20,29,30)}$. Otra limitación podría ser la pérdida de una unidad experimental del grupo de moringa en la mitad del experimento. El presente es un estudio preclínico que comprueba efectos hipoglicemiantes en animales de experimentación, pero deben realizarse estudios en seres humanos que prueben estos beneficios y midan otros riesgos. Asimismo, por cuestiones logísticas, no se estudió aspectos de la fisiopatología de acción del aloxano y de los tratamientos, así como la caracterización de los fitoquímicos en los extractos.

Tabla 3. Comparación de la glicemia al octavo día de tratamiento con $200 \mathrm{mg} / \mathrm{kg}$ PV de M. oleifera, $140 \mathrm{mg} / \mathrm{kg}$ PV de S. sonchifolius y metformina, en ratas con diabetes inducida

\begin{tabular}{lcccc}
\hline Grupo & $\mathbf{n}$ & Mediana & Valor de $\mathbf{p}^{\star}$ & Comparación $^{* *}$ \\
\hline Control & 6 & 586,5 & & $\mathrm{~A}$ \\
Metformina & 6 & 126,5 & & $\mathrm{~B}$ \\
M. oleifera & 5 & 246,0 & 0,015 & $\mathrm{~B}$ \\
S. sonchifolius & 6 & 134,0 & & $\mathrm{~B}$ \\
\hline
\end{tabular}

*Valor de p de Kruskall Wallis

${ }^{*}$ Letras iguales indican grupos con datos similares (test de Dunn). 
Tabla 4. Comparación de la glicemia al décimo quinto día de tratamiento con $200 \mathrm{mg} / \mathrm{kg}$ PV M. oleifera, $140 \mathrm{mg} / \mathrm{kg}$ PV de S. sonchifolius y metformina, en ratas con diabetes inducida.

\begin{tabular}{lcccc}
\hline Grupo & n & Mediana & Valor de $\mathbf{p}^{*}$ & Comparación $^{* *}$ \\
\hline Control & 6 & 389,0 & & $\mathrm{~A}$ \\
Metformina & 6 & 107,0 & & $\mathrm{~B}$ \\
M. oleifera & 5 & 110,0 & 0,004 & $\mathrm{~B}$ \\
S. sonchifolius & 6 & 116,5 & & $\mathrm{~B}$ \\
\hline
\end{tabular}

* Valor de p de Kruskall Wallis.

${ }^{*}$ Letras iguales indican grupos con datos similares (test de Dunn).

Se concluye que el extracto acuoso de Smallanthus sonchifolius a $140 \mathrm{mg} / \mathrm{kg}$ PV y de Moringa oleifera a $200 \mathrm{mg} / \mathrm{kg}$ PV, y metformina a $14 \mathrm{mg} / \mathrm{kg}$ PV no presentaron diferencias significativas en su efecto hipoglicemiante sobre ratas con diabetes inducidas con aloxano, a los ocho y 15 días de tratamiento. Por ello,

\section{REFERENCIAS BIBLIOGRÁFICAS}

1. Organización Mundial de la Salud. Diabetes [Internet]. OMS; 2016. [citado el 25 de marzo de 2018]. Disponible en: http://www.who.int/ mediacentre/factsheets/fs312/es/.

2. Organización Mundial de la Salud. Día Mundial de la Salud 2016: diabetes [Internet]. OMS; 2016. [citado el 25 de marzo de 2018]. Disponible en: http://www.who.int/campaigns/world-health-day/2016/event/es/.

3. Asociación Latinoamericana de Diabetes. Guías ALAD sobre el diagnóstico, control y tratamiento de la Diabetes Mellitus Tipo 2 con medicina basada en evidencia. 2013. Revista de la Asociación Latinoamericana de la Salud [Internet]. ALAD; 2013 [citado el 30 de marzo de 2018]. Disponible en: https://issuu.com/alad-diabetes/docs/guias_alad_2013.

4. Instituto Nacional de Estadística e Informática del Perú. Perú: Enfermedades no transmisibles y transmisibles, 2019 [Internet]. INEI; 2020 [citado el 10 de junio de 2020]; p. 33. Disponible en: https://proyectos.inei.gob. pe/endes/2019/SALUD/ENFERMEDADES_ENDES_2019.pdf.

5. Instituto Nacional de Estadística e Informática del Perú. En el Perú 3 de cada 100 personas de 15 y más años reportan tener diabetes [Internet]. INEI; 2016 [citado 29 de marzo de 2018]. Disponible en: https://www.inei.gob.pe/prensa/noticias/en-el-peru-3-de-cada-100personas-de-15-y-mas-anos-reportan-tener-diabetes-8993/.

6. Omodanisi E, Aboua Y, Oguntibeju O. Assessment of the Anti-Hyperglycaemic, Anti-Inflammatory and Antioxidant Activities of the Methanol Extract of Moringa Oleifera in Diabetes-Induced Nephrotoxic Male Wistar Rats. Molecules. 2017;22(4):439. doi: 10.3390/ molecules22040439.

7. Guzmán-Gómez G, Arce A, Saavedra H, Rojas M, Solarte J, Mina M, et al. Adherencia al tratamiento farmacológico y control glucémico en pacientes adultos con diabetes mellitus tipo 2. Alad. 2018;8(1):35-43. doi: 10.24875/ALAD.18000319.

8. Association American Diabetes. Pharmacologic approaches to glycemic treatment: standards of medical care in diabetes-2018. Diabetes Care. 2018;41(1):S73-85. doi: 10.2337/dc18-S008.

9. Russo D, Valentão P, Andrade PB, Fernandez EC, Milella L. Evaluation of antioxidant, antidiabetic and anticholinesterase activities of Smallanthus sonchifolius landraces and correlation with their phytochemical profiles. Int J Mol Sci. 2015;16(8):17696-718. doi: 10.3390/ ijms160817696.

10. Shah M, Keach J, Panichayupakaranant P. Antidiabetic naphthoquinones and their plant resources in Thailand. Chem Pharm Bull. 2018;66(5):483-92. doi: 10.1248/cpb.c17-00529. se recomienda realizar estudios complementarios de seguridad, donde se evalúe toxicidad y citotoxicidad, in vitro e in vivo, y posteriormente realizar estudios clínicos para comprobar si el efecto hipoglicemiante encontrado en el presente estudio es similar en seres humanos. También se recomienda estudiar interacciones y sinergias entre los tratamientos, así como la fisiopatología de sus efectos.

Contribuciones de los autores: DMSM, OWVT, JPAT y LZBG concibieron y diseñaron el manuscrito; HSD analizó e interpretó los datos; DMSM, OWVT y HSD redactaron el manuscrito. DMSM, OWVT, HSD y LZBG participaron en la recolección de resultados. Todos los autores participaron en la revisión crítica del artículo, aprobación de la versión final y asumen responsabilidad frente a los contenidos del artículo.

Fuentes de financiamiento: Autofinanciado.

Conflictos de interés: Los autores no tienen ningún conflicto de interés que declarar.
11. Inocente-Camones M, Guija-Poma E, Zarzosa-Norabuena E, Loja Herrera B, Ponce-Pardo J. Efecto hipoglicemiante de los extractos acuoso y etanólico de Psidium guajava L. (Guayaba) en ratas diabéticas inducidas por aloxano. Horiz Med. 2015;15(2):41-48.

12. Castañeda B, Castro de la Mata R, Manrique R, Ibánez L, Fujita R, Barnett, et al. Estudio fitoquímico y farmacológico de 4 plantas con efecto hipoglicemiante. Horiz Med. 2008;8(1):6-34.

13. Ahmad J, Khan I, Blundell R. Moringa oleifera and glycemic control: A review of current evidence and possible mechanisms. Phytotherapy research. 2019;33(11):2841-8. doi: 10.1002/ptr.6473.

14. Chávez J, Carvallo T, González A. Identificación de compuestos agonistas a receptores involucrados en el síndrome metabólico de un extracto de hoja de Moringa oleifera. Centro de Investigación en Alimentación y Desarrollo. 2016;1(1):6.

15. Padilla F, Cruz J. Extractos de hojas de Moringa oleífera en la prevención y tratamiento de la diabetes mellitus. Revista Cubana de Medicina Natural y Tradicional. 2018;2(1).

16. Yassa H, Tohamy A. Extract of Moringa oleifera leaves ameliorates streptozotocin-induced Diabetes mellitus in adult rats. Acta Histochemica. 2014;116(5):844-54. doi: 10.1016/j.acthis.2014.02.002.

17. de Almeida Paula HA, Abranches MV, de Luces Fortes Ferreira CL. Yacon (Smallanthus sonchifolius): a food with multiple functions. Crit Rev Food Sci Nutr. 2015;55(1):32-40. doi: 10.1080/10408398.2011.645259.

18. Gordillo G, Negrón L, Zúñiga T, Flores E, Moreyra R, Fuertes C, et al. The hypoglycemic effect of aqueous extract of leaves of Smallanthus sonchifolius (yacon) in patients with Type 2 Diabetes Mellitus. Ciencia e Investigación. 2014;15(1):42-7.

19. Serra-Barcellona C, Habib N, Honoré S, Sánchez S, Genta S. Enhydrin Regulates Postprandial Hyperglycemia in Diabetic Rats by Inhibition of a-Glucosidase Activity. Plant Foods Hum Nutr. 2017;72(2):156-60. doi: 10.1007/s11130-017-0600-y.

20. Barreto S, Báez S, Malvetti V, Cardozo M, Gill A, Matto J, et al. Moringa oleifera acute effect on dexamethasone-induced hyperglycemia in Wistar rats. An Fac Cienc Méd. 2016;48(1):41-8. doi: 10.18004/ anales/2015.048(01)41-048.

21. Rodriguez J, Soplapuco C. Efecto del Smallanthus sonchifolius sobre los niveles de glucosa e insulina plasmática en Oryctalagus cuniculis con diabetes inducida con alloxano [Tesis para optar el título de Médico Cirujano]. Lambayeque: Universidad Nacional Pedro Ruiz Gallo; 2004.

22. Justil C, Angulo P, Justil H, Arroyo J. Evaluación de la actividad hipoglicemiante del extracto acuoso de Abuta grandifolia (Mart.) en ratas 
con diabetes inducida por aloxano. Rev Inv Vet Perú. 2015;26(2):20612. doi: 10.15381/rivep.v26i2.11008.

23. ISO 15197:2013 In vitro diagnostic test systems - Requirements for blood-glucose monitoring systems for self-testing in managing diabetes mellitus [Internet]. ISO; 2013 [citado 6 de junio de 2018]. Disponible en: https://www.iso.org/standard/54976.html.

24. Unión Europea. Directiva 2010/63/UE del Parlamento Europeo y del Consejo, de 22 de septiembre de 2010, relativa a la protección de los animales utilizados para fines científicos [Internet]. UE; 2010. Disponible en: http:// www.madrid.org/rlma_web/html/web/FichaNormativa.icm?ID=1942.

25. Dos Santos KC, Bueno BG, Pereira LF, Francisqueti FV, Braz MG, Bincoleto LF, et al. Yacon (Smallanthus sonchifolius) leaf extract attenuates hyperglycemia and kkeletal muscle oxidative stress and inflammation in diabetic rats. Evid Based Complement Alternat Med. 2017;2017(9):6418048. doi: 10.1155/2017/6418048.

26. Mejía A, Zuloeta D, Palacios F. Efecto hipoglucemiante del consumo de yacón (Smallantus sonchifolius) en ratones diabéticos tipo 2 inducidos con aloxano. Revista Científica de Ciencias de la Salud. 2016;9(1):72-8. doi: $10.17162 /$ rccs.v9i1.549.

27. Fahey JW, Wade KL, Stephenson KK, Shi Y, Liu H, Panjwani AA, et al. A Strategy to deliver precise oral doses of the glucosinolates or isothiocyanates from Moringa oleifera leaves for use in clinical studies. Nutrients. 2019;11(7):1547. doi: 10.3390/nu11071547.

28. Abd El Latif A, El Bialy BES, Mahboub HD, Abd Eldaim MA. Moringa oleifera leaf extract ameliorates alloxan-induced diabetes in rats by regeneration of $\beta$ cells and reduction of pyruvate carboxylase expression. Biochem Cell Biol. 2014;92(5):413-9. doi: 10.1139/bcb2014-0081.

29. Idakwoji P, Barnabas A, Elah S. Co-administration of ethanolic leaf extract of Moringa oleifera and metformin reverses polyphagia, polydipsia and stabilizes body weight in alloxan-induced diabetic rats. Biokemistri. 2015;27(3):129-38.

30. Habib N, Serra-Barcellona C, Honoré S, Genta S, Sánchez S. Yacon roots (Smallanthus sonchifolius) improve oxidative stress in diabetic rats. Pharm Biol. 2015;53(8):1183-93. doi: 10.3109/13880209.2014.970285. 